\title{
Severe asthma in pediatrics: Outcomes of the implementation of a special health care protocol
}

\author{
Verónica Giubergia, M.D. ${ }^{a}$, María J. Ramirez Farías, M.D. ${ }^{a}$, Verónica Pérez, M.D. ${ }^{a}$, \\ Adriana González, B.S. ${ }^{b}$, Nancy Crespi, B.S. ${ }^{a}$, Nora Fridman, B.S. ${ }^{c}$ and \\ Claudio Castaños, M.D. ${ }^{a}$
}

\begin{abstract}
Introduction. Severe asthma accounts for $5-7 \%$ of all asthma cases. The World Health Organization proposed a follow-up protocol to classify cases into severe, treatment-resistant asthma (STRA) or severe, difficult-to-control asthma (SDCA).

Objective. To analyze the clinical, functional, and therapeutic characteristics of patients with STRA or SDCA.

Methods. Cross-sectional, observational, and analytical study to assess the diagnosis, the extent of control (clinical and functional), comorbidities, treatment adherence, inhalation technique, and environmental factors in patients with severe asthma.
\end{abstract}

Results. A total of 69 patients were included: STRA $(n=33)$ and SDCA $(n=36)$. In the group withSTRA, $100 \%$ of patients had been previously the group with SDCA ( $p=0.03)$. In the group with STRA, $63 \%$ required admission to the intensive care unit (ICU); $82 \%$, ventilatory support; and 1 patient, tracheostomy. In the group with SDCA, $54 \%$ required admission to the ICU; and $33 \%$, ventilatory support $(p=0.03)$.

The baseline spirometry was normal in theSDCA group; a mild obstructive ventilatory defect was observed in the STRA group ( $p=0.009$ ).

In the SDCA group, treatment adherence was lower $(p=0.01)$. Higher inhaled corticosteroid doses were required in the STRA group $(p=0.0001)$. Omalizumab was indicated in the case of STRA $(p=0.0001)$. After 6 months of follow-up, more than $75 \%$ of children in both groups achieved asthma control.

Conclusions. A significant lack of treatment adherence was observed in the SDCA group. In this group, treatment doses were reduced. Asthma was controlled in a high percentage of children with STRA and SDCA.

Key words: severe asthma, treatment resistance, prevention and control, program.

http: / / dx.doi.org/10.5546/ aap.2018.eng.105

E-mail address:

Verónica Giubergia, M.D.

verogiubergia@gmail. com

Funding:

None.

Conflict of interest:

None.

Received: 4-21-2017

Accepted: 9-5-2017

To cite: Giubergia V, Ramirez Farías MJ, Pérez V, et al. Severe asthma in pediatrics: Outcomes of the implementation of a special health care protocol. Arch Argent Pediatr 2018;116(2):105-111. hospitalized due to asthma compared to $87.8 \%$ in

\section{GLOSSARY}

ACT: Asthma Control Test. ERS / ATS: European Respiratory Society / American Thoracic Society. FEF: forced expiratory flow between $25 \%$ and $75 \%$ of the forced vital capacity.

$\mathrm{FEV}_{1} / \mathrm{FVC}$ : forced expiratory volume in 1 second and forced vital capacity ratio.

$\mathrm{FEV}_{1}$ : forced expiratory volume in 1 second.

FVC: forced vital capacity.

HRCT: high-resolution computed tomography.

ICSs: inhaled corticosteroids.

ICU: intensive care unit.

LABAs: long-acting beta ${ }_{2}$-agonists.

MVS: mechanical ventilatory

support.

PANAG: Health Care Program

for Children with Problematic

Severe Asthma (Programa de

Atención de Niños con Asma Grave

Problemática).

SA: severe asthma.

SDCA: severe, difficult-to-control asthma

STRA: severe, treatment-resistant asthma.

VS: ventilatory support.

WHO: World Health Organization.

\section{INTRODUCTION}

Asthma is the most common chronic disease during childhood and the main cause of pediatric morbidity. ${ }^{1}$ There are approximately 300000000 people with asthma, and the highest prevalence is observed in the pediatric age group. Argentina ranks at an intermediate level: $16.4 \%$ in children aged 6-7 years and $10.9 \%$ in children aged 13-14 years. ${ }^{1,2}$ 
In our country, asthma causes more than 400 deaths (10\% in patients aged 5-39 years) and more than 15000 hospitalizations every year. ${ }^{1,3}$

These occur in patients with more severe asthma, especially uncontrolled asthma cases. Severe asthma (SA) accounts for $5-7 \%$ of all asthma cases. They make up a small, highly vulnerable group. ${ }^{1,4,5}$ They often have asthma attacks and are hospitalized, and are at risk for developing adverse events due to highdose corticosteroid therapy. These children use emergency services 15 times more and are hospitalized 20 times more than those with mild or moderate asthma. ${ }^{6}$

In recent years, the World Health Organization (WHO) proposed a protocol for the follow-up of patients with uncontrolled SA that assessed different factors that caused morbidity, thus facilitating classification and treatment. This strategy helped to classify asthma into severe, difficult-to-control asthma (SDCA) due to modifiable causes, with an adequate clinical course, or into severe, treatment-resistant asthma (STRA), with a more complex clinical course and treatment. . $^{7-10}$

The implementation of interdisciplinary health care strategies for the follow-up of patients with SA has demonstrated their effectiveness. ${ }^{6,11}$ Since 2008, the Department of Pulmonology of Hospital Garrahan has offered a Health Care Program for Children with Problematic Severe Asthma (Programa de Atención de Niños con Asma Grave, PANAG) with the participation of pulmonologists, nurses, physical therapists, and psychologists and a special health care modality that includes frequent visits, provision of medications at no charge, development of educational activities, etc. ${ }^{6}$

The objective of this study was to analyze the clinical, functional, and therapeutic characteristics of patients with STRA or SDCA who completed follow-up in a public hospital in accordance with a special health care protocol.

\section{MATERIAL AND METHODS}

This was a cross-sectional, observational, and analytical study that analyzed the outcomes of implementing a health care protocol in patients receiving follow-up in the PANAG. Children were admitted to the program if they had failed to control symptoms with high-dose inhaled corticosteroids (ICSs) (problematic asthma), attended the Department of Pulmonology or the Emergency Department for assessment every month or every two months or, in the case of exacerbations, every day. ${ }^{6}$

This study was conducted between June and December 2016 and included all male and female children aged 6-18 years receiving follow-up in the program who had presumptive diagnosis of SA according to the Global Initiative for Asthma classification: daytime symptoms, frequent exacerbations, usual nocturnal symptoms, limited physical activity, and high-dose ICS requirements ( $\geq 800 \mu \mathrm{g}$ of budesonide or a similar drug) and beta $_{2}$-agonists (LABAs) to maintain asthma control. ${ }^{4}$ Patients with cystic fibrosis, obliterative bronchiolitis or bronchopulmonary dysplasia were excluded.

Patients were assessed by an interdisciplinary team according to the WHO protocol to differentiate those with STRA from those with SDCA, and received follow-up in the same program after their participation. The protocol included reassessment during 6 months after diagnosis, extent of asthma control, factors associated with lack of control (comorbidities, lack of treatment adherence, incorrect inhalation technique, and environmental factors), and treatment adjustment ${ }^{6-9}$ (Figure 1). These aspects were considered during assessment, which included 6 visits.

SDCA was defined as cases for whom factors were detected once the diagnosis was confirmed, such as technique and/or adherence errors according to the treating team assessment, difficulties to access medication or emotional disorders, and who managed to control asthma once these factors were corrected. STRA was defined as cases who, after considering modifiable factors, did not achieve an adequate asthma control (daytime symptoms $>2$ times per week, nocturnal awakenings, salbutamol $>2$ times per week, limitation of physical activity) and required high-dose treatment (ICSs, LABAs, montelukast) and, eventually, omalizumab or oral corticosteroids to achieve an adequate asthma control. ${ }^{1,4,5}$

Comorbidities (rhinitis, sinusitis, gastroesophageal reflux, obesity) were assessed in both groups. An esophageal pH-metry or impedance analysis was requested by the gastroenterologist. If an alternative diagnosis was suspected (e.g., obliterative bronchiolitis) or other concurrent diseases (e.g., bronchiectasis) were suspected, a chest and sinus high-resolution computed tomography (HRCT) was done and then assessed by a radiologist. In all cases, a 
sweat test, human immunodeficiency virus (HIV) serology, immunoglobulin dosage, and purified protein derivative (PPD) were done. Children with a body mass index $\geq 95^{\text {th }}$ percentile were considered obese. ${ }^{12}$ Patients with associated diseases were referred to a specialist for followup and treatment.

Treatment adherence and the inhalation technique were assessed in all visits. Adherence was assessed by an interdisciplinary team, who estimated the total number of doses the patient had to receive in the specific period between appointments and the remaining number of doses in the inhaler dose counter.

Recommendations on environmental control (smoking at home and household environmental contaminants), the technique, and treatment adherence were discussed during workshops for parents and children (recreational activities, physical exercise, physical therapy training on normal respiratory patterns, and relaxation techniques for self-control) coordinated by psychologists and physical therapists. ${ }^{6}$

Pulmonary function was assessed in all visits using a Medgraphics spirometer (MGC Diagnostics). Spirometries were done to analyze forced vital capacity (FVC), forced expiratory volume in 1 second $\left(\mathrm{FEV}_{1}\right), \mathrm{FEV}_{1} / \mathrm{FVC}$ ratio, and forced expiratory flow between $25 \%$ and $75 \%$ of the FVC (FEF $25 / 75 \%$ ) in accordance with the American Thoracic Society/European Respiratory Society (ATS/ERS) criteria. ${ }^{13} \mathrm{~A}$ baseline spirometry and one done after 6 months were analyzed. All outcome measures were expressed in percentages.

Asthma control was assessed using the Asthma Control Test (ACT). Values $\geq 20$ indicated controlled asthma. ${ }^{14}$ The result of the ACT test done after 6 months of follow-up was included in this study.

The objectives of this study were explained to patients, parents or legal tutors, who gave their consent for participation.

\section{STATISTICS}

Value distribution within each outcome was described by means of measures of central tendency and scattered plots or categorically. Categorical outcome measures were compared using the $\chi^{2}$ test. Continuous outcomes were compared with the Student's t test or the Wilcoxon test. $P$ values $\leq 0.05$ were considered significant. The Stata XIII software (Stata-Corp, College Station, TX) was used.

\section{RESULTS}

A total of 69 children who were receiving follow-up in the PANAG were included in the study; $45 \%(\mathrm{n}=31)$ were boys; and their mean age was 12.4 years (standard deviation [SD]: 4.1). Besides, $48 \%(n=33)$ were defined as having SDCA and 52\% ( $\mathrm{n}=36)$, STRA. The characteristics of patients are described in Table 1.

All children with STRA $(n=36)$ had been hospitalized for asthma prior to follow-up initiation, whereas $87.8 \%(n=29)$ of patients with

FIGURE 1. Clinical spectrum of severe asthma in pediatrics ${ }^{7,8}$

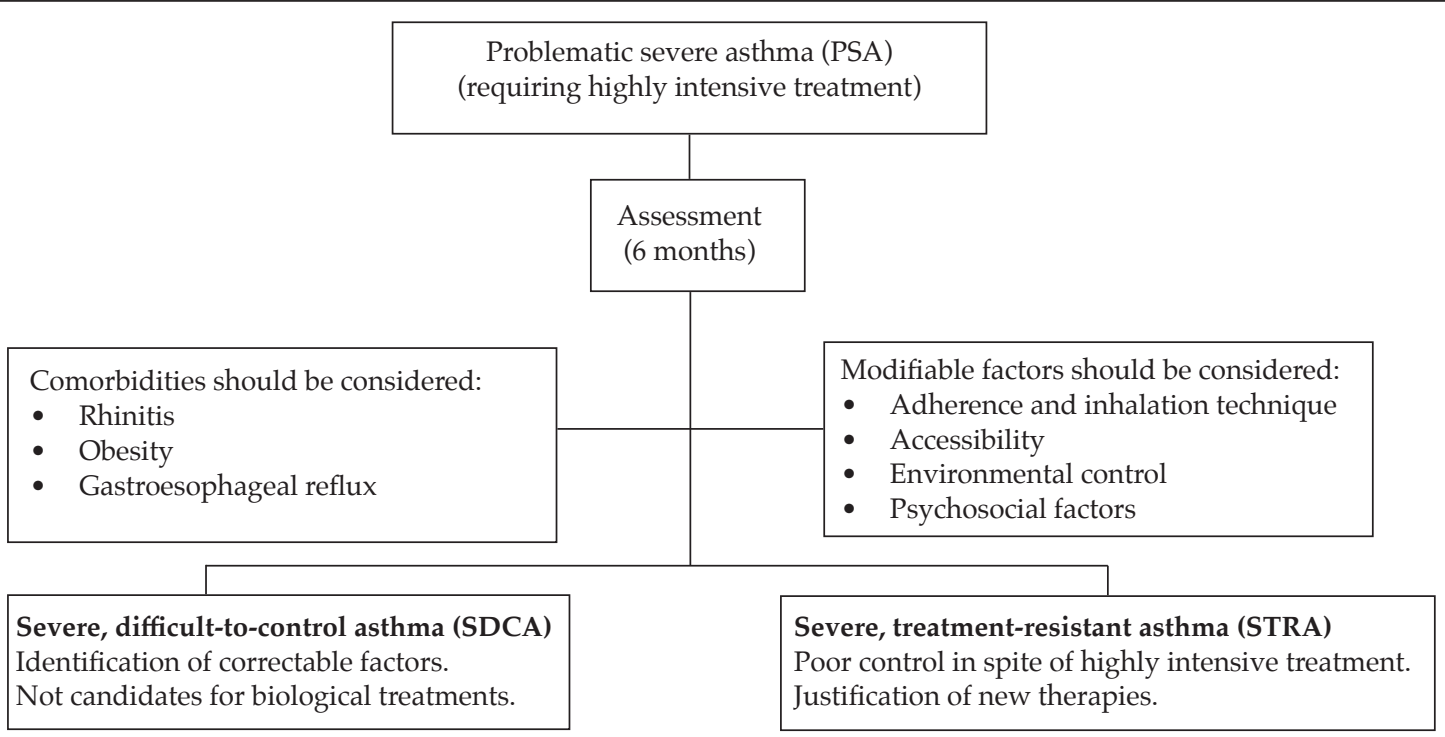


SDCA had required hospitalization $(p=0.03)$. Among patients with STRA, 63\% $(n=23)$ required admission to the intensive care unit (ICU); of these, $82 \%(\mathrm{n}=19)$ required ventilatory support (VS) and 1 patient, a tracheostomy. However, in the SDCA group, $54 \%$ of patients $(n=18)$ required ICU admission and only 33\% ( $\mathrm{n}=11)$, VS $(p=0.03)$.

Comorbidities, immunoglobulin E (IgE), and blood eosinophilia were similar in both groups. A higher number of patients from the SDCA group did not adhere to treatment $(p=0.01)$ (Table 1).

A chest HRCT was done in 30 patients with STRA (83.3\%), compared to only 6 patients $(18 \%)$ from the SDCA group $(p=0.0001)$. Findings included air trapping, laminar atelectasis, and peribronchial cuffing. Small regions of bronchiectasis were observed in the medium lobe of 2 patients with STRA.

The STRA group showed mild obstructive ventilatory disorder, evidenced only as a reduction in the $\mathrm{FEV}_{1} / \mathrm{FVC}$ ratio, unlike patients from the SDCA group, who had a normal baseline spirometry $(p=0.009)$. After 6 months, a significant increase was observed in all parameters analyzed in both groups (Table 2).

Also after 6 months of follow-up, the STRA group remained on significantly higher ICS and LABA doses than the SDCA group $(p=0.0001$ and $p=0.006$ ). A higher number of patients required montelukast in the STRA group and omalizumab

TABLE 1. Characteristics of children diagnosed with severe, difficult-to-control asthma $(n=33)$ and severe, treatment-resistant asthma $(n=36)$

\begin{tabular}{|c|c|c|c|}
\hline Outcome measures & $\begin{array}{l}\text { ere, difficult-to-control asthma } \\
448 \%(\mathrm{n}=33)\end{array}$ & $\begin{array}{l}\text { Severe, treatment-resistant asthma } \\
\qquad 52 \%(\mathrm{n}=36)\end{array}$ & P value \\
\hline Sex (male) & $48.3 \%(n=15)$ & $51.6 \%(n=16)$ & 0.5 \\
\hline Age (years old) & $13.7(12.2-15)^{*}$ & $11.2(10-12.5)^{*}$ & 0.01 \\
\hline Age at symptoms onset (months old) & $2.2(1.4-3)^{*}$ & $1(0.6-1.4)^{*}$ & 0.02 \\
\hline Family history of asthma & $52 \%(\mathrm{n}=15)$ & $48 \%(\mathrm{n}=14)$ & 0.6 \\
\hline Serum IgE (IU / mL) & $904(558-1249)^{*}$ & $1249(1045-1433)^{*}$ & 0.7 \\
\hline Eosinophilia & $452(332-572)^{*}$ & $522(338-706)^{*}$ & 0.5 \\
\hline Rhinitis & $48 \%(\mathrm{n}=16)$ & $41.6 \%(\mathrm{n}=15)$ & 0.6 \\
\hline Obesity & $21 \%(\mathrm{n}=7)$ & $11 \%(\mathrm{n}=4)$ & 0.2 \\
\hline Gastroesophageal reflux & $15 \%(\mathrm{n}=5)$ & $11 \%(\mathrm{n}=4)$ & 0.06 \\
\hline Smokers at home & $39 \%(\mathrm{n}=13)$ & $33.3 \%(\mathrm{n}=12)$ & 0.6 \\
\hline No treatment adherence & $86 \%(\mathrm{n}=28)$ & $56 \%(n=20)$ & 0.01 \\
\hline
\end{tabular}

IgE: immunoglobulin E.

${ }^{*}$ Values are expressed as average and confidence interval $(95 \% \mathrm{CI})$

TABLE 2. Pulmonary function in children with severe, difficult-to-control asthma $(n=33)$ and severe, treatment-resistant asthma $(n=36)$ at follow-up initiation and after 6 months

\begin{tabular}{|c|c|c|c|}
\hline Outcome measures & $\begin{array}{l}\text { Severe, difficult-to-control asthma } \\
448 \%(\mathrm{n}=33)\end{array}$ & $\begin{array}{l}\text { Severe, treatment-resistant asthma } \\
\qquad 52 \%(\mathrm{n}=36)\end{array}$ & $P$ value \\
\hline Initial FVC & $94.7 \%(89.5-100)$ & $99 \%(93-105)$ & 0.2 \\
\hline Final FVC & $98.8 \%(93-103)$ & $105.7 \%(101-109.7)$ & 0.05 \\
\hline P value & 0.04 & 0.01 & \\
\hline 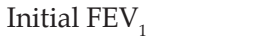 & $89.7 \%(89-95)$ & $87 \%(80-94)$ & 0.5 \\
\hline Final $\mathrm{FEV}_{1}$ & $93.9 \%(89-98)$ & $98.4 \%(93-104)$ & 0.1 \\
\hline Pvalue & 0.04 & 0.0001 & \\
\hline Initial $\mathrm{FEV}_{1} / \mathrm{FVC}$ & $85.5(82-88)$ & $79(75-83)$ & 0.009 \\
\hline Final $\mathrm{FEV}_{1} / \mathrm{FVC}$ & $84(81-86)$ & $83(80-86)$ & 0.6 \\
\hline Pvalue & 0.2 & 0.003 & \\
\hline Initial FEF $25 / 75 \%$ & $81.8 \%(72-91)$ & $74.5 \%(60-88)$ & 0.3 \\
\hline Final FEF $25 / 75 \%$ & $88.4 \%(76-99)$ & $85.7 \%(75-96)$ & 0.7 \\
\hline P value & 0.05 & 0.02 & \\
\hline
\end{tabular}

Values are expressed as average and confidence interval (95\% CI).

FVC: forced vital capacity; FEV1: forced expiratory volume in 1 second; FEV1/FVC: forced expiratory volume in 1 second and forced vital capacity ratio; FEF 25/75\%: forced expiratory flow between 25\% and 75\% of the forced vital capacity.

The Student's test was used to analyze results between groups. The Student's test was used to analyze paired data at follow-up initiation and after 6 months in the same group. 
was the only drug indicated in this group $(p=0.05$ and $p=0.0001$ ) (Table 3). After 6 months of followup, 75\% $(\mathrm{n}=25)$ of patients from the SDCA group and $80 \%(n=25)$ from the STRA group achieved an adequate asthma control $(p=0.6)$.

\section{DISCUSSION}

This study described the characteristics of a cohort of patients diagnosed with problematic SA and receiving follow-up as part of a special health care protocol in a public hospital. After 6 months under assessment, more than $50 \%$ of cases were defined as STRA. For the rest of the patients, interventions aimed at modifiable factors allowed to reclassify them as SDCA once treatment was adjusted in a timely manner.

Establishing the difference between the STRA group patients, whose symptoms remained uncontrolled in spite of receiving the highest recommended treatment regimen or whose adequate control could only be maintained on high doses, and the SDCA group patients, whose asthma could be controlled after eliminating reversible factors, is a challenge. ${ }^{8,9}$ Few studies have been published that assessed these populations. ${ }^{15,16}$

To this end, the WHO proposed a protocol to facilitate the follow-up of patients with uncontrolled SA. ${ }^{78}$ Initially, the protocol defines the diagnosis of problematic SA. ${ }^{7,8}$ These children have persistent symptoms and/or severe exacerbations in spite of receiving high-dose ICSs. ${ }^{8-10}$ They require a systematic assessment: diagnosis confirmation, careful assessment of treatment adherence and inhalation technique, adequate management of comorbidities, and environmental control. Following this strategy, $50-75 \%$ of these children will have SDCA caused by potentially modifiable factors, as the ones mentioned above. Their clinical course is favorable with available treatments. The remaining $25-50 \%$ will be made up of patients with STRA, the most severe form of asthma, for whom more specific treatments are considered. Both groups require regular monitoring. ${ }^{16,17}$

In this study, during each visit, the treating team systematically assessed the inhalation technique and worked on treatment adherence, environmental control, and the patient's housing conditions. These interventions were implemented individually and in groups through workshops for parents and children. ${ }^{6}$

Differences between groups were observed. Symptom onset started early in life among children with STRA, which was consistent with published data. ${ }^{18}$ All patients had been hospitalized due to asthma and more than half required admission to the ICU and / or VS, whereas a significantly lower-although relevantnumber of patients from the SDCA group had been hospitalized, which evidenced the severity of asthma in both groups.

In this series, as in others, baseline $\operatorname{IgE}$ or eosinophilia levels were not considered parameters that distinguished between groups. ${ }^{15,16}$ The frequency of gastroesophageal reflux, rhinitis or obesity, and the presence of smokers at home, were similar to other published series, and no differences were observed between groups. ${ }^{15}$

In adults with SA, pulmonary function is usually affected. In pediatrics, even in patients with STRA, the spirometry may be normal or show a mild obstruction. ${ }^{18-20}$ However, some authors have suggested that pulmonary function in pediatrics would allow to distinguish between STRA and SDCA, contrary to what other authors have stated. ${ }^{15,16,21}$ In this study, only children with STRA had a mild but significant obstruction at follow-up initiation, evidenced by a reduced $\mathrm{FEV}_{1} / \mathrm{FVC}$ ratio which then returned to normal. After 6 months, both groups showed significant improvements in all spirometry parameters. Treatment optimization resulted in an improved pulmonary function, regardless of normal or

TABLE 3. Treatment given to patients diagnosed with severe, difficult-to-control asthma $(n=33)$ and severe, treatmentresistant asthma $(n=36)$ after 6 months of follow-up

\begin{tabular}{|c|c|c|c|}
\hline Outcome measures & $\begin{array}{l}\text { evere, difficult-to-control asthma } \\
\qquad 48 \%(n=33)\end{array}$ & $\begin{array}{l}\text { Severe, treatment-resistant asthma } \\
\qquad 52 \%(\mathrm{n}=36)\end{array}$ & P value \\
\hline Inhaled corticosteroids ( $\mu \mathrm{g} /$ day) & $601(509-693)^{*}$ & $836(762-910)^{*}$ & 0.0001 \\
\hline LABAs ( $\mu \mathrm{g} /$ day) & $56(46-67.3)^{*}$ & $77(66.8-87.4)^{*}$ & 0.006 \\
\hline Montelukast & $12 \%(n=4)$ & $30 \%(\mathrm{n}=11)$ & 0.05 \\
\hline Oral methylprednisolone & $0 \%(\mathrm{n}=0)$ & $5.5 \%(\mathrm{n}=2)$ & 0.1 \\
\hline Omalizumab & $0(\mathrm{n}=0)$ & $41.6 \%(\mathrm{n}=15)$ & 0.0001 \\
\hline
\end{tabular}

* Values are expressed as average and confidence interval (95\% CI). LABAs: long-acting beta ${ }_{2}$-agonists. 
almost normal baseline values in both groups, which evidenced the method's lack of sensibility to detect the extent of airway inflammation. It is striking that patients with an almost normal baseline spirometry may go on without an adequate asthma control. A hypothesis that may account for the mild changes observed in the $\mathrm{FEV}_{1} / \mathrm{FVC}$ ratio among patients with frequent symptoms is the increased bronchomotor tone and its lability. Anyhow, the presence of histopathological changes, such as bronchial restructuring, in schoolchildren and adolescents, cannot be ruled out. ${ }^{18,22,23}$

An adequate classification of cases into SDCA or STRA not only minimizes unnecessary tests and procedures, it also reduces the risks for adverse drug reactions. ${ }^{16}$ Asthma control with lower doses and fewer drugs is possible in children with SDCA. However, patients with STRA may not achieve an adequate asthma control, even once potential risk factors are eliminated. These patients require higher ICS doses and are candidates for new therapeutic strategies, such as monoclonal antibodies (omalizumab) or continuous oral corticosteroids. ${ }^{8,9,17,24}$ In this series, patient classification allowed to individualize treatments. A high percentage of children with SDCA achieved symptom control with significantly lower ICS / LABA doses and fewer patients required montelukast compared to the STRA group. Only in this group, patients were treated with omalizumab or oral corticosteroids.

The costs related to SA may incur up to $29 \%$ of the family income. ${ }^{15}$ In this study, access was warranted because both the drugs and metered dose inhalers were provided by the hospital at no charge so as to control a major cause of lack of treatment adherence, especially in a population with limited financial resources. ${ }^{25}$

Another major factor associated with inadequate control was poor treatment adherence. Multiple reasons lie behind the lack of treatment adherence, including costs, complex treatment regimens, risk for side effects, and lack of parental monitoring, among others. ${ }^{25,26}$ Although there is no completely reliable method to verify adherence, the recommendation is to monitor and promote compliance with therapeutic indications using any method available at the time of consult. Successful adherence is defined as the administration of at least $80 \%$ of prescribed doses. However, only $24 \%$ of patients with SDCA achieve treatment adherence $\geq 80 \%$, whereas $41 \%$ of children with STRA comply with prescribed treatments. In this study, a high percentage of patients with SDCA failed to adhere to the prescribed treatment. However, considering that parent-reported adherence is usually higher than actual adherence, lack of treatment adherence in both groups was alarming but consistent with other publications. ${ }^{16}$

The prevalence of SA is very low (5\% of all asthma cases). In this series, a high number of patients with SA were assessed and received an adequate follow-up as part of a special health care program, which facilitated data collection. However, the observational nature of this study did not completely exclude potential confounding factors.

\section{CONCLUSION}

In this population, the early onset of symptoms and a history of hospitalization in the ICU with VS requirement were characteristics significantly present in patients with STRA compared to those with SDCA. In addition, implementing a health care protocol helped to determine that lack of treatment adherence was the most significantly observed modifiable factor in the SDCA group, which allowed to significantly reduce ICS doses in these children. A high percentage of patients from both groups achieved asthma control, thus confirming the relevance of this strategy.

\section{REFERENCES}

1. Comité Nacional de Neumonología; Comité Nacional de Alergia; Comité Nacional de Medicina Interna; et al. Guía de diagnóstico y tratamiento: asma bronquial en niños $\geq 6$ años.Actualización 2016. Resumenejecutivo. Arch Argent Pediatr 2016;114(6):595-6.

2. Mallol J, Crane J, von Mutius E, et al. The International Study of Asthma and Allergies in Childhood (ISAAC) Phase Three: a global synthesis. Allergol Immunopathol (Madr) 2013;41(2):73-85.

3. Argentina. Ministerio de Salud. Secretaria de Políticas, Regulación e Institutos. Dirección de Estadísticas e Información de Salud. Estadísticas Vitales: Información básica año 2013. [Accessed on: September $8^{\text {th }}, 2017$ ]. Available at: http: / / www.bvs.org.ar/pdf/anuario13.pdf.

4. Global Strategy for Asthma Management and Prevention. Global Initiative for Asthma (GINA) 2016. [Accessed on: September $8^{\text {th }}$, 2017]. Available at: http: / / ginasthma.org/ wp-content/uploads / 2016/04/GINA-2016-main-report_ tracked.pdf.

5. British guideline on the management of asthma. Anational clinical guideline October 2014;141. British Thoracic Society. [Accessed on: September $\left.8^{\text {th }}, 2017\right]$. Available at: https: / / www.brit-thoracic.org.uk/document-library/clinicalinformation/asthma/btssign-asthma-guideline-2014/.

6. Giubergia V, Fridman N, González Pena H. Evaluación del impacto de un programa de atención de niños con asma grave. Arch Argent Pediatr 2012;110(5):382-7.

7. Bush A, Zar HJ. WHO universal definition of severe asthma. Curr Opin Allergy Clin Immunol 2011;11(2):115-21. 
8. Bush A, Hedlin G, de Benedictis F, et al. Severe childhood asthma: a common international approach? Lancet 2008;372(9643);1019-21.

9. Hedlin G, Bush A, LødrupCarlsen K, et al. Problematic severe asthma in children, not one problem but many: a GA2LEN initiative. Eur Respir J 2010;36(1):196-201.

10. BushA,PedersenS,Hedlin G, etal.Pharmacological treatment of severe, therapy-resistant asthma in children: what can we learn from where? Eur Respir J 2011;38(4):947-58.

11. Brandão HV, Cruz CMS, Santos Ida S Jr, et al. Hospitalizations for asthma: impact of a program for the control of asthma and allergic rhinitis in Feira de Santana, Brazil. J Bras Pneumol 2009;35(8):723-9.

12. Centers for Diseases Control and Prevention. National Center for Health Statistics. National Health and Nutrition Examination Survey. Anthropometric Reference Data, United States 1988-1994. [Accessed on: September $8^{\text {th }}$, 2017]. Available at: http://www.cdc.gov/nchs/about/ major/nhanes / Anthropometric\%20Measures.htm.

13. Miller MR, Hankinson J, Brusasco V, et al. Standardization of spirometry. Eur Respir J 2005;26(2):319-38.

14. Rodrigo GJ, Arcos JP, Nannini LJ, et al. Reliability and factor analysis of the Spanish version of the asthma control test. Ann Allergy Asthma Immunol 2008;100(1):17-22.

15. DeAndrade WC, Lasmar LM, RicciCdeA, et al. Phenotypes of severe asthma among children and adolescents in Brazil: a prospective study. BMC Pulm Med 2015;15:36.

16. CookJ, Beresford F, Fainardi V, et al. Managing the pediatric patient with refractory asthma: a multidisciplinary approach. J Asthma Allergy 2017;10:123-30.
17. Martin Alonso A, Saglani S. Mechanisms Mediating Pediatric Severe Asthma and Potential Novel Therapies. Front Pediatr 2017;5:154.

18. Rodrigues AM, Roncada C, Santos G, et al. Clinical characteristics of children and adolescents with severe therapy-resistant asthma in Brazil. J Bras Pneumol 2015;41(4):343-50.

19. Jenkins HA, Cherniack R, Szefler SJ, et al. A comparison of the clinical characteristics of children and adults with severe asthma. Chest 2003;124(4):1318-24.

20. Lang AM, Konradsen J, Carlsen $\mathrm{KH}$, et al. Identifying problematic severe asthma in the individual child - does lung function matter? Acta Paediatr 2010;99(3):404-10.

21. Konradsen JR, Nordlund B, Lidegran M, et al. Problematic severe asthma: a proposed approach to identifying children who are severely resistant to therapy. Pediatr Allergy Immunol. 2011;22(1 Pt 1):9-18.

22. Saglani S, Payne DN, Zhu J, et al. Early detection of airway wall remodeling and eosinophilicinflammation in preschool wheezers. Am J Respir Crit Care Med 2007;176(9):858-64.

23. Payne DN, Rogers AV, Adelroth E, et al. Early thickening of the reticular basement membrane in children with difficult asthma. Am J RespirCrit Care Med 2003;167(1):78-82.

24. Neffen H, Vidaurreta S, Balanzat A, et al. Asma de difícil control en niños y adolescentes: estrategias diagnósticoterapéuticas. Medicina (B Aires) 2012;72(5):403-13.

25. Anderson W, Szefler S. New and future strategies to improve asthma control in children. J Allergy Clin Immunol2015;136(4):848-59.

26. Bourdin A, Halimi L, Vachier I, et al. Adherence in severe asthma. Clin Exp Allergy 2012;42(11):1566-74. 